\title{
Understanding and Improving SIGCHI's Volunteer Experience
}

Rojin Vishkaie, Microsoft, Monica Pereira, London Metropolitan University, Mark Perry, Brunel University London, George E. Raptis, Human Opsis, Heloisa Candello, IBM Research, Ceara Byrne, Georgia Institute of Technology

Interactions, Volume 27, Issue 5, page 80, https://doi.org/10.1145/3416524

Volunteering underpins SIGCHI's work, but volunteer efforts are coming under huge stress across SIGCHI's venues and activities-at conferences, supporting local SIGs, and in its various committees. The most obvious pressure lies in scaling to conference growth and the increase in publishing output while the number of reviewers grows at a slower rate. Expanding the volunteer pool is an obvious, if not easily resolvable, solution. In addition, the human and systems processes for working with and managing our amazing volunteers could be reorganized to make more effective use of them. To meet this latter goal, the SIGCHI Executive Committee decided to create a new position, adjunct chair for volunteer development, which will look closely at why and how volunteering is done. This AC recently convened a Volunteer Development Committee. The committee has already identified issues around the volunteer selection process, which has not always been as transparent or inclusive as it could be.

\section{Why do people volunteer?}

Lisa Thomas, Gary Pritchard, and Pam Briggs [1] say there are four implicit promises of volunteering: 1) social (e.g., social network and community building), 2) opportunity (e.g., improved skills, new employment opportunities, and personal development), 3) value (e.g., more meaningful use of free time aligned with religious, political, social, or ideological beliefs), and 4) organizational (e.g., organizational citizenship, job satisfaction, reward, and recognition). All of these are likely to have an impact on any new support systems that we offer.

\section{What is the Volunteer Development Committee roadmap?}

The SIGCHI Volunteer Development Committee has developed a three-stage project roadmap to address the stresses we currently face. In 2019, during the first stage of the project, we planned a broad survey and interview-based study of volunteering for SIGCHI venues and activities. The survey solicits a wide range of perceptions and experiences, while identifying the differences in volunteer problems, barriers, opinions, needs, and expectations from the diverse groups in the SIGCHI community. The interviews will develop a detailed understanding of work that individuals involved in volunteer recruitment and management do, and reflect on how this impacts on the wider SIGCHI community and its values. They will help identify the scope and scale of experienced and anticipated problems, identify potential opportunities for change, and review new technical systems for supporting their work.

We are now in the second stage of the project, conducting the surveys and interviews. Once our analysis is completed, we will present the findings to the SIGCHI community for feedback. This process will iteratively continue to inform and evaluate the design specification for a new volunteer-management system (supporting onboarding, user profiles and achievements, conflicts of interest, advertising volunteer opportunities, and providing educational content). In the third stage, we plan to implement and evaluate this volunteering system in real-life contexts to assess its effectiveness, both in supporting SIGCHI volunteers and in its impact on SIGCHI venues where volunteering has been under strain.

As part of its larger community effort, the Volunteer Development Committee also offers support to the diverse set of SIGCHI venues and groups in providing guidance, guidelines, training, and policies around volunteer recruitment, and exploring potential implications arising from the European General Data Protection Regulation (GDPR).

\section{Endnote}

1. Thomas, L., Pritchard, G., and Briggs, P. Digital design considerations for volunteer recruitment: Making the implicit promises of volunteering more explicit. Proc. of C\&T 2019; https://doi.org/ $10.1145 / 3328320.3328368$ 